\title{
Neural basis of the cognitive map: Path integration does not require hippocampus or entorhinal cortex
}

\author{
Yael Shrager*, C. Brock Kirwan ${ }^{\dagger}$, and Larry R. Squire ${ }^{* \$ \$ \uparrow||}$ \\ Departments of *Neurosciences, "Psychiatry, and \$Psychology and †'Institute for Neural Computation, University of California at San Diego, \\ La Jolla, CA 92093; and ๆVeterans Affairs Healthcare System, San Diego, CA 92161
}

Contributed by Larry R. Squire, June 4, 2008 (sent for review May 23, 2008)

The hippocampus and entorhinal cortex have been linked to both memory functions and to spatial cognition, but it has been unclear how these ideas relate to each other. An important part of spatial cognition is the ability to keep track of a reference location using self-motion cues (sometimes referred to as path integration), and it has been suggested that the hippocampus or entorhinal cortex is essential for this ability. Patients with hippocampal lesions or larger lesions that also included entorhinal cortex were led on paths while blindfolded (up to $15 \mathrm{~m}$ in length) and were asked to actively maintain the path in mind. Patients pointed to and estimated their distance from the start location as accurately as controls. A rotation condition confirmed that performance was based on self-motion cues. When demands on long-term memory were increased, patients were impaired. Thus, in humans, the hippocampus and entorhinal cortex are not essential for path integration.

amnesia | medial temporal lobe | memory | spatial cognition | navigation

$\mathrm{F}$ or several decades, two influential ideas have been central to discussions about the function of the hippocampus, entorhinal cortex, and related medial temporal lobe structures. One perspective emphasizes the importance of these structures for memory $(1,2)$, and the other emphasizes their importance for spatial cognition (3-5). An important aspect of spatial cognition is the ability to keep track of a reference location during movement using internal cues (i.e., self-motion cues), sometimes referred to as path integration $(3,4)$. Yet, keeping track of a reference location requires memory. Accordingly, it has been unclear how proposals about memory and proposals about spatial cognition relate to each other.

The view that medial temporal lobe structures are important for memory makes a key distinction between what is referred to as short-term (or working) memory and long-term memory. Working memory (i.e., the ability to hold information actively in mind [e.g., a short list of digits]) is independent of medial temporal lobe structures (6-8), whereas long-term memory is critically dependent on these structures. Accordingly, patients with hippocampal or entorhinal damage should perform poorly on memory tasks only when demands are made on long-term memory. If a task could be performed within the span of working memory instead, then patients should succeed despite damage to the hippocampus or entorhinal cortex. This idea applies even to tasks that require spatial cognition, such as path integration.

The view that medial temporal lobe structures are important for spatial cognition grew out of the finding that the rat hippocampus contains place cells, cells that exhibit activity specific to an animal's location in space (9). In addition, grid cells were recently discovered in rat entorhinal cortex, upstream from hippocampal place cells. Grid cells exhibit a grid-like structure of place fields that repeat at regular intervals across the environment, suggesting that major steps in computing spatial location information occur in entorhinal cortex, immediately afferent to the hippocampus $(10,11)$. These findings raise the possibility that the hippocampus and entorhinal cortex might be required to accomplish path integration. If so, a question arises about the performance of patients with damage to the hippocampus and entorhinal cortex in those cases when the path integration task can be managed within working memory. On the one hand, the patients might perform well, as they do in other tasks that can be supported by working memory. On the other hand, the patients might be impaired at path integration, and the distinction between working memory and long-term memory might not be relevant. That is, medial temporal lobe structures might be needed to carry out the computations that support path integration. We have tested these possibilities by asking whether the hippocampus and entorhinal cortex are essential for keeping track of a reference location even when the task can be managed within the span of working memory.

\section{Results}

Condition 1: Standard. Participants were led on 16 different paths (Fig. 1), and at the end of each path, they were asked to point to their start location (mean trial duration $=33.4 \mathrm{sec}$ ). Circular statistics (12) revealed that both groups exhibited a significant (Moore's test, $P<0.05$ ) and similar (rank-sum test, $P>0.1$ ) pointing direction (controls $=4^{\circ}$, patients $=-4^{\circ}$, Fig. $2 a$ ) and that the pointing direction for each group did not differ from the correct direction $\left(0^{\circ}\right)$ ( $\mathrm{V}$ test, $P$ values $\left.>0.1\right)$. The dispersion of individual mean scores (i.e., the extent to which the individual means in each group clustered around that group's mean) was also similar for controls and patients (nonparametric test for dispersion, $P>0.1$ ). Further, each participant exhibited a significant pointing direction. Notably, the two patients with large lesions that included all the hippocampus and entorhinal cortex (E.P. and G.P.) exhibited pointing directions that were well within control range (E.P. $=-10^{\circ}$ and G.P. $=-7^{\circ}$, control range: $-14^{\circ}$ to $\left.+20^{\circ}\right)$.

To quantify the variability within individual participants, we next averaged for each group the SDs of the 16 pointing responses made by each individual. Fig. $2 c$ shows that the individual variability of controls and patients was nearly identical (controls $=30.5$, patients $=31.3)$. The variability of patients E.P. and G.P. was well within control range (E.P. $=40.6$ and G.P. $=$ 37.0, control range: $14.0-66.6)$. To determine whether participants were in fact engaged in path integration, we asked the two most severely memory-impaired patients (E.P. and G.P.) and four controls immediately after they pointed how they had accomplished the task. All subjects uniformly described trying to keep track of their position in space as they moved, continually updating their position relative to the start point. There was no hint that anyone tried to do postwalk calculations of any kind.

\footnotetext{
Author contributions: Y.S., C.B.K., and L.R.S. designed research; Y.S. and C.B.K. performed research; Y.S. and C.B.K. analyzed data; and Y.S., C.B.K., and L.R.S. wrote the paper. The authors declare no conflict of interest.

\|To whom correspondence should be addressed. E-mail: Isquire@ucsd.edu.

This article contains supporting information online at www.pnas.org/cgi/content/full/ 0805414105/DCSupplemental.

(C) 2008 by The National Academy of Sciences of the USA
} 

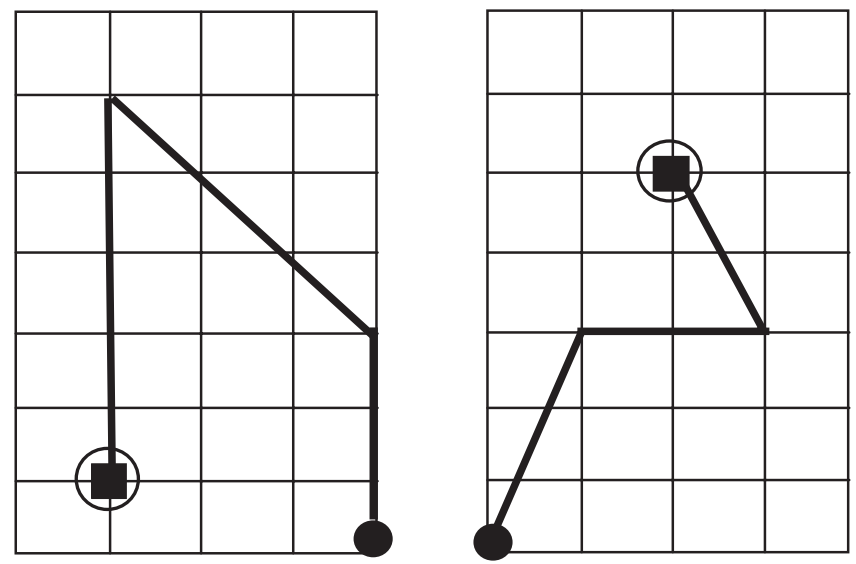

Fig. 1. Sample routes. In each of five conditions, blindfolded participants were led in an indoor 2.4-m $\times$ 4.3-m area (conditions 1, 3, 4, and 5) or in an outdoor $5-\mathrm{m} \times 15-\mathrm{m}$ open area (condition 2 ) along paths that ended at a circular platform (small circle around filled squares; there was not a platform in condition 2). In conditions 1, 3, 4, and 5, half the routes involved one turn and half involved two turns. In condition 2 , all routes involved two turns. Filled circles, start; filled squares, finish. In conditions 1 and 2 (16 and 8 trials, respectively), participants pointed to the start location shortly after stepping onto the platform (mean interval from start $=33.4$ and $29.7 \mathrm{sec}$, respectively). In condition 3 (8 trials), participants walked a path and, shortly after stepping onto the platform, estimated their distance from the start location (mean interval from start $=32.1 \mathrm{sec}$ ). In condition 4 (16 trials), participants walked a path, stepped onto the platform, and then pointed to the start location after being rotated $190^{\circ}$ at $14^{\circ} / \mathrm{sec}$ (mean interval from start $=32.4 \mathrm{sec}$ ). In condition 5 (16 trials), participants walked a path, stepped onto the platform, and then pointed to the start location after being engaged in an unrelated task of mental navigation (mean interval from start $=1 \mathrm{~min} 10 \mathrm{sec}$ ).

Condition 2: Longer Paths. In the standard condition, we showed that path integration could be accomplished despite damage to the hippocampus or the hippocampus plus entorhinal cortex. We next asked whether path integration might be impaired if the task were more demanding, albeit still manageable within working memory. The two patients with large medial temporal lobe lesions (E.P. and G.P.) and four controls were led in an outdoor space on eight paths that were nearly four times longer than the paths in condition $1(15 \mathrm{~m}$ in this condition vs. $4.3 \mathrm{~m}$ in condition 1 ) and that more closely resembled a natural walk. The mean trial time was $29.7 \mathrm{sec}$. The mean pointing direction and individual variability for controls were $9^{\circ}$ and 35.0 (Fig. $2 b$ and $d$ ). For E.P. and G.P., the mean pointing direction was $-17^{\circ}$ and $-13^{\circ}$, and the individual variability was 24.3 and 29.9, respectively. Both patients were well within the range of the controls with respect to both the absolute value of the pointing direction (range $=$ $-15^{\circ}$ to $+34^{\circ}$ ) and individual variability (24.1-47.0).

Condition 3: Distance. In a third condition, we tested the ability of participants to estimate the distance between the start location and the end location. The five patients and seven controls who participated in condition 1 made similar estimates. For the four shorter paths $($ mean $=1.6 \mathrm{~m})$, patients estimated a distance of $1.9 \mathrm{~m}$ and controls estimated a distance of $1.5 \mathrm{~m}$; for the four longer paths $($ mean $=4.0 \mathrm{~m})$, patients estimated a distance of $2.9 \mathrm{~m}$ and controls estimated a distance of $2.7 \mathrm{~m}$ ( $t$ test values $<1.1, P$ values $>0.3$ ). Further, for each group, the estimates for the four shorter distances were smaller than the estimates for the four longer distances ( $t$ test values $>4.0, P$ values $<0.02$ ).

Condition 4: Rotation. In another condition (rotation), we tested whether participants were in fact performing path integration by using internal cues rather than by relying on external cues beyond experimental control (mean trial length $=32.4 \mathrm{sec}$ ). Pilot
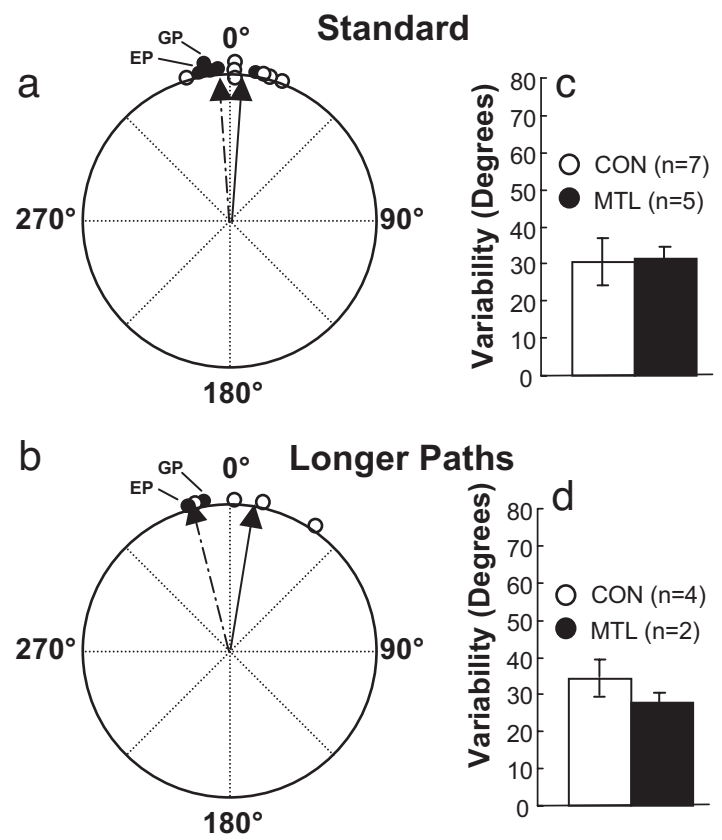

Fig. 2. Circular means of each participant's $16(a)$ or $8(b)$ pointing directions in conditions 1 and 2, respectively, for patients with damage to the medial temporal lobe (MTL, filled circles) and controls (CON, unfilled circles). The correct direction is indicated by $0^{\circ}$. Group pointing directions are also indicated (solid arrow $=$ CON; broken arrow $=\mathrm{MTL}$ ). Shorter arrows denote greater variability (dispersion) in the group's pointing direction (following Moore's test for nonuniformity (12). The SD of pointing directions around each participant's circular mean was calculated $(c, d)$, and the individual SDs were then averaged for each group (individual variability). Brackets indicate standard error.

experiments indicated that during rotation, participants had difficulty knowing how far they had been turned. We therefore expected that if participants were in fact relying on path integration (internal cues) to point to their starting location, then they would have difficulty when a rotation was introduced into the standard condition (condition 1 ). The results confirmed that performance was substantially compromised in the rotation condition. First, neither group exhibited a significant pointing direction (Moore's test, $P$ values $>0.1$, Fig. $3 a$ ). Second, for both groups, the variability of each individual's performance was markedly increased as compared with the standard condition (again, measured as the SD of each individual's 16 pointing
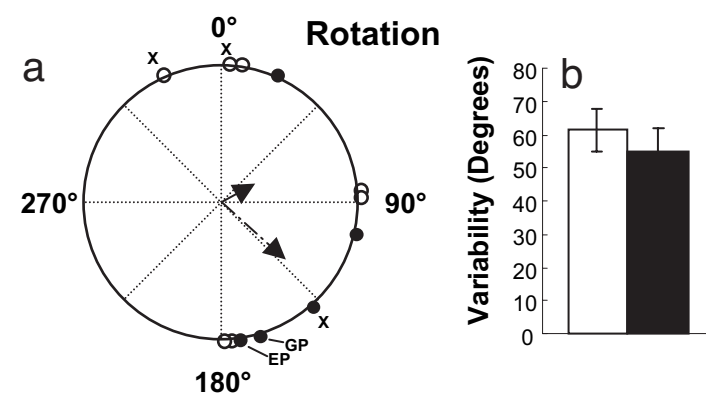

Fig. 3. Rotation. (a) Circular means of each participant's 16 pointing directions in condition 4 (rotation) for patients with damage to the medial temporal lobe (MTL, filled circles) and controls (CON, unfilled circles). The correct direction is indicated by $0^{\circ}$. Group pointing directions are also indicated (solid arrow = CON; broken arrow = MTL). " $X$ " indicates individuals who did not exhibit a significant point direction. (b) Individual variability for each group is shown. Brackets indicate standard error. 


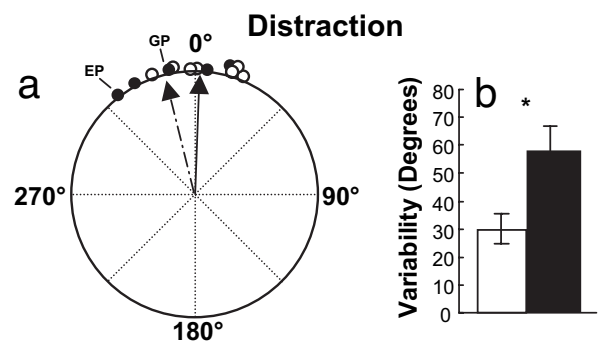

Fig. 4. Distraction. (a) Circular means of each participant's 16 pointing directions in condition 5 (delay and distraction) for patients with damage to the medial temporal lobe (MTL, filled circles) and controls (CON, unfilled circles). The correct direction is indicated by $0^{\circ}$. Group pointing directions are also indicated (solid arrow $=\mathrm{CON}$; broken arrow $=\mathrm{MTL}$ ). (b) Individual variability for each group is shown. Asterisk $\left(^{*}\right)$ indicates $P<0.05$. Brackets indicate standard error.

directions: controls $=61.5$ in the rotation condition vs. 30.5 in the standard condition, patients $=54.9$ vs. $31.3, t$ test values $>3.1, P$ values $<0.04$, Fig. $3 b)$. Third, the marked variability in individual performance was similar for the two groups (61.5 vs. 54.9, $\left.t_{(10)}<0.7, P>0.5\right)$.

Condition 5: Delay and Distraction. In a final condition (distraction), we increased the long-term memory demands of the task by increasing the duration of each trial (modeled after the trials in the standard condition) and by introducing distraction during the longer delay (total trial length $=1 \mathrm{~min} 10 \mathrm{sec}$ ). The controls performed as well in the distraction condition as in the standard condition (mean pointing direction $=1^{\circ}$ in the distraction condition vs. $4^{\circ}$ in the standard condition) (Fig. $4 a$ ). As in the standard condition, controls also had a significant pointing direction (Moore's test, $P<0.05$ ) that was not different from $0^{\circ}$ (V test, $P>0.1$ ). Further, for the control group, the variability of individual pointing directions in the distraction condition (30.1) was no greater than the variability of individual pointing directions in the standard condition (30.5) (compare Fig. $4 b$ with Fig. $2 c)$. In contrast, the patients had difficulty in the distraction condition. On the one hand, they did exhibit a significant pointing direction (Moore's test, $P<0.05$; mean pointing direction $=-14^{\circ}$ in the distraction condition vs. $-4^{\circ}$ in the standard condition) that was not different from $0^{\circ}$ (V test, $P>$ 0.1 ) and not different from the mean pointing direction of controls $\left(-14^{\circ}\right.$ vs. $1^{\circ}$, rank-sum test, $\left.P>0.1\right)$. On the other hand, by this measure, five of the six patients performed more poorly in the distraction condition than in the standard condition. For example, E.P.'s pointing direction was quite poor $\left(-42^{\circ}\right.$, compared with $-10^{\circ}$ in the standard condition). More importantly, distraction dramatically increased the variability of individual patient performance across the 16 trials (57.1 in the distraction condition vs. 31.3 in the standard condition, $t>2.9, P<0.05$, compare Fig. $4 b$ with Fig. $2 c$ ). E.P.'s variability was 77.0, G.P.'s was 67.4 , and both values were outside the range of controls (16.7-58.0). Further, an ANOVA of the individual variability scores for the standard and distraction conditions revealed a Group X Condition interaction $(F>11.0, P<0.01)$, indicating that the patients were more affected by distraction than the controls.

To illustrate more dramatically the severity of memory impairment in E.P. and G.P., we asked them several minutes after testing to describe paths they had walked and to describe the task they had been engaged in. Neither patient could remember anything of what they had been doing and suggested they had been "in conversation" (E.P.) or "looking at objects" (G.P.). These observations make it clear that when E.P. and G.P. succeeded in the navigation tasks described here, they succeeded by maintaining the start location in working memory.

\section{Discussion}

We have shown that patients with lesions limited to the hippocampus and patients with larger lesions that include the entorhinal cortex can keep track of a reference location as well as controls on paths up to $15 \mathrm{~m}$ in length and involving one or two turns. After being led on a path, and while being deprived of external cues, the patients pointed as accurately as the controls to their start location. They also estimated as accurately as the controls the distance between the start and end locations. The performance of both groups was disrupted in the rotation condition, indicating that participants were using self-motion cues and not using any external cues in the environment. Finally, introducing a long-term memory requirement to the task impaired the performance of the patients.

By intention, the paths used in our study were relatively short (involving one or two turns and a duration of less than $35 \mathrm{sec}$ ) so that they might be maintained within working memory. It is possible that an impairment would have been detected if we had not been limited by the memory impairment of the patients and had been able to test much more complex paths. Still, if the hippocampus and entorhinal cortex were essential for path integration (we refer to path integration as the ability to calculate information about the distance from and the direction to a reference point), one would have expected the patients to have some difficulty as soon as their paths involved turning and moving across a reasonable distance. Instead, we found that performance was entirely intact for paths as long as $15 \mathrm{~m}$ that involved up to two turns.

An earlier study found that one of the patients studied here (E.P.), who has extensive medial temporal lobe damage that includes all the hippocampus and entorhinal cortex, performed well when asked to mentally navigate through the environment where he grew up and also to point toward locations in that environment when imagining himself facing a direction specified by the experimenter (13). Thus, the ability to mentally navigate an environment is also intact when the spatial environment was learned long before the brain damage occurred.

Our data are difficult to reconcile with the view that the hippocampus and entorhinal cortex are essential sites for the computations necessary to keep track of a reference location using self-motion cues. There is no doubt that cells with distinct spatial properties are found in both the hippocampus and entorhinal cortex $(9-11)$ and that these cells can represent detailed spatial information in addition to many other relevant features of ongoing behavioral episodes in service of memory function (14). A recent study using functional MRI found that hippocampal activation correlated with path integration accuracy in healthy volunteers (15). We suggest that damage to the hippocampus would not impair performance on this task so long as the task did not exceed the span of working memory. Our findings are not inconsistent with the possibility that the computations needed for path integration are carried out in parallel at more than one site (including the medial temporal lobe), but the findings rule out the idea that the medial temporal lobe is the only site that can carry out these computations.

Our study assesses the effects of bilateral hippocampal and entorhinal damage on path integration by untangling the spatial demands of the task from its potential demands on long-term memory. A few studies have examined the effect of lesions on path integration ability but have yielded mixed results. In one study, patients with right temporal lobe lesions who were led along a path were impaired at estimating direction (but not distance) information (16). However, in these cases, the lateral temporal lobe was extensively damaged (inferior and middle temporal gyrus). In another study, rats with hippocampal lesions 
exhibited normal path integration ability (17). In two other studies, rats with lesions of the hippocampus or entorhinal cortex were impaired at path integration $(18,19)$. None of the rodent studies reported the time needed to accomplish each trial, although it seems likely that the trials in some cases may have been relatively short. Still, the possibility remains that these tasks placed demands on long-term memory in rats. Additionally, it is possible that there are substantive species differences between humans and rodents, such that the more developed neocortex in humans might be capable of supporting path integration, whereas in rodents, the hippocampus and entorhinal cortex might be more important.

Our data support the view that medial temporal lobe structures are important for long-term memory and not for the spatial computations needed for path integration, so long as performance can be supported by working memory. It is possible that path integration is accomplished in parallel at more than one site (e.g., in the medial temporal lobe and also in the parietal cortex), with the result that damage to the medial temporal lobe would leave path integration intact. Alternatively, the computations necessary for path integration may be carried out upstream of the medial temporal lobe, perhaps in the parietal cortex (3). Thus, damage to the parietal cortex impairs performance on a variety of spatial tasks in rats, monkeys, and humans (20-22), including path integration (19). Further, cells exhibiting activity specific to a particular path (a sequence of left and right turns through an environment) have been found in rat parietal cortex (23). By this view, spatial information from the cortex arrives at the medial temporal lobe, like information from other modalities (e.g., visual information, auditory information), and the medial temporal lobe then carries out the operation of transforming perception into long-term memory.

\section{Methods}

Participants. Five memory-impaired patients (mean age $=66$ years, one female) and seven matched controls (mean age $=69$ years, two female) were tested for their path integration ability. Two patients are profoundly amnesic and have large well-characterized lesions of the medial temporal lobe, including all the hippocampus, all the entorhinal cortex, all the perirhinal cortex, and the majority of the parahippocampal cortex (E.P. and G.P) [see supporting information (SI)]. These patients have demonstrated virtually no new learning since the onset of their amnesia, and during repeated testing over many weeks, they do not recognize that they have been tested before (24). Three patients are moderately amnesic and have well-characterized lesions limited to the hippocampus (K.E., L.J., and G.W.) [see supporting information $(S /)]$.

Condition 1: Standard. Participants wore a blindfold and noise-canceling earphones, and verbal instructions were transmitted through the earphones. Participants were led on 16 paths (8 involving one turn, 8 involving two turns) in a $2.4-\mathrm{m} \times 4.3-\mathrm{m}(8-\mathrm{ft} \times 14-\mathrm{ft})$ space (Fig. 1). Mean path length was $4.3 \mathrm{~m}$ $(14.2 \mathrm{ft})$. Because the patients have impaired long-term memory, we intended to use paths short enough that they might be actively maintained in mind (i.e., they should not exceed working memory capacity) and could be traversed in less than $1 \mathrm{~min}$. Participants were encouraged to actively maintain the paths in mind during each trial. Ensuring that the task could be performed within the span of working memory was essential so that the memory-impaired patients would not be disadvantaged by their long-term memory deficits.

At the end of each path, participants stepped onto a platform raised $5 \mathrm{~cm}$

1. Scoville WB, Milner B (1957) Loss of recent memory after bilateral hippocampal lesions. I Neurol Neurosurg Psychiatry 20:11-21.

2. Squire LR, Stark CE, Clark RE (2004) The medial temporal lobe. Annu Rev Neurosci 27:279-306.

3. Etienne AS, Jeffery KJ (2004) Path integration in mammals. Hippocampus 14:180-192.

4. McNaughton BL, Battaglia FP, Jensen O, Moser El, Moser MB (2006) Path integration and the neural basis of the "cognitive map." Nat Rev Neurosci 7:663-678.

5. O'Keefe J, Nadel L (1978) The Hippocampus as a Cognitive Map (Clarendon, Oxford, UK).

6. Atkinson RC, Shiffrin RM (1968) The Psychology of Learning and Motivation: Advances in Research and Theory (Academic, New York). above the floor and equipped with handlebars to ensure the stability of the participants. After a short delay (10-17 sec), participants were asked to point to their start location (mean trial length $=33.4 \mathrm{sec}$ ). Two independent raters measured the direction in which participants pointed (measurements were taken to the nearest degree from a grid beneath the platform, mean interrater error $=4^{\circ}$ ). The pointing direction was then recorded in degrees for each trial, where $0^{\circ}$ indicated perfect performance. For each participant, we derived the circular mean (mean pointing direction) and a measure of variability across the 16 trials. On each trial, participants began in a different start location, and the path ended in a different location. Participants were blindfolded at the start location but before the platform was moved to the next end location. Further, the handlebars were always in line with the final path direction taken by the participant, and thus did not provide information about where the path started.

Condition 2: Longer Paths. The two patients with large medial temporal lobe lesions (E.P. and G.P.) and four controls were given a test of path integration using longer paths (Fig. 1). Participants again wore a blindfold and noisecanceling earphones, and they were led on eight different paths in an outdoor open space. Each path involved two turns, and traversal of the path resembled a natural walk (path length $=15 \mathrm{~m}$ ). At the end of the path, participants used handlebars for support (held in place by one of the experimenters) and pointed to their start location (mean trial length $=29.7 \mathrm{sec}$ ).

Condition 3: Distance. The same 12 participants as in condition 1 wore a blindfold and earphones and were led on eight paths, similar to those in the standard condition (four involving one turn, four involving two turns). Half the paths (two involving one turn, two involving two turns) ended only a short distance from the start location (mean $=1.6 \mathrm{~m}$ ), and half the paths (two involving one turn, two involving two turns) ended a longer distance from the start $($ mean $=4.0 \mathrm{~m})$. At the end of the path, participants stepped onto the platform; after a short delay (similar in length to the delay in condition 1), they were asked to estimate in feet the distance between their current location and the start location. The mean trial length was $32.1 \mathrm{sec}$.

Condition 4: Rotation. The 12 participants from condition 1 again wore a blindfold and earphones and were led to the platform along 16 new paths (mirror images of the paths in the standard condition). Immediately after they stepped onto the platform, a remotely controlled motor within the platform slowly rotated the participant for a distance of $190^{\circ}$ at a low speed $\left(\approx 14^{\circ} / \mathrm{sec}\right.$ ). Pilot experiments indicated that at this rotation speed, participants had difficulty knowing how far they had been rotated. Mean trial length matched that of the standard condition $(32.4 \mathrm{sec})$.

Condition 5: Delay and Distraction. All participants (from condition 1) wore a blindfold and earphones and were led on 16 paths (the same as in the standard condition but in a different order). Immediately after stepping onto the platform, participants were instructed to remain stationary while engaging in one to three tasks of mental navigation. For each task, they were first asked to imagine themselves facing an initial heading direction (north, south, east, or west). They then carried out mentally a sequence of three instructions (e.g., turn $90^{\circ}$ right and take a step, turn $90^{\circ}$ left and take a step, turn $90^{\circ}$ left and take a step). They then reported their final heading direction (north, south, east, or west). (For results, see SI). At the end of this filled delay, participants pointed to the start location of the path. The average trial length was $1 \mathrm{~min}$ $10 \mathrm{sec}$.

ACKNOWLEDGMENTS. We thank Jennifer Frascino and Christine Smith for assistance, and Edvard Moser and Wendy Suzuki for their helpful comments. This work was supported by the Medical Research Service of the Department of Veterans Affairs, the National Institute of Mental Health Grant MH24600, the Metropolitan Life Foundation, and a National Science Foundation Predoctoral Fellowship (to Y.S.).

7. Drachman DA, Arbit J (1966) Memory and the hippocampal complex. II. Is memory a multiple process? Arch Neurol 15:52-61.

8. Shrager Y, Levy DA, Hopkins RO, Squire LR (2008) Working memory and the organization of brain systems. J Neurosci 28:4818-4822.

9. O'Keefe J, Dostrovsky J (1971) The hippocampus as a spatial map. Preliminary evidence from unit activity in the freely-moving rat. Brain Res 34:171-175.

10. Fyhn M, Molden S, Witter MP, Moser El, Moser MB (2004) Spatial representation in the entorhinal cortex. Science 305:1258-12564.

11. Hafting T, Fyhn M, Molden S, Moser MB, Moser El (2005) Microstructure of a spatial map in the entorhinal cortex. Nature 436:801-806.

12. Batschelet E (1981) Circular Statistics in Biology (Academic, London). 
13. Teng E, Squire LR (1999) Memory for places learned long ago is intact after hippocampal damage. Nature 400:675-677.

14. Wood ER, Dudchenko PA, Eichenbaum H (1999) The global record of memory in hippocampal neuronal activity. Nature 397:613-616.

15. Wolbers T, Wiener JM, Mallot HA, Buchel C (2007) Differential recruitment of the hippocampus, medial prefrontal cortex, and the human motion complex during path integration in humans. J Neurosci 27:9408-9416.

16. Worsley CL, et al. (2001) Path integration following temporal lobectomy in humans. Neuropsychologia 39:452-464.

17. Alyan S, McNaughton BL (1999) Hippocampectomized rats are capable of homing by path integration. Behav Neurosci 113:19-31.

18. Maaswinkel H, Jarrard LE, Whishaw IQ (1999) Hippocampectomized rats are impaired in homing by path integration. Hippocampus 9:553-561.
19. Parron C, Save E (2004) Evidence for entorhinal and parietal cortices involvement in path integration in the rat. Exp Brain Res 159:349-359.

20. Kolb B, Walkey J (1987) Behavioural and anatomical studies of the posterior parieta cortex in the rat. Behav Brain Res 23:127-145.

21. Mesulam MM (1981) A cortical network for directed attention and unilateral neglect. Ann Neurol 10:309-325.

22. Save E, Moghaddam M (1996) Effects of lesions of the associative parietal cortex on the acquisition and use of spatial memory in egocentric and allocentric navigation tasks in the rat. Behav Neurosci 110:74-85.

23. Nitz DA (2006) Tracking route progression in the posterior parietal cortex. Neuron 49:747-756.

24. Bayley PJ, Frascino JC, Squire LR (2005) Robust habit learning in the absence of awareness and independent of the medial temporal lobe. Nature 436:550-553. 